\title{
Alterstice
}

Revue internationale de la recherche interculturelle

International Journal of Intercultural Research

Revista International de la Investigacion Intercultural

\section{L'exploitation d'un corpus d'interactions en tandem anglais/français pour mieux comprendre les enjeux de la rétroaction corrective entre pairs}

\section{Céline Horgues et Sylwia Scheuer}

Volume 8, numéro 1, 2018

Jumelage interculturel et pédagogie universitaire

URI : https://id.erudit.org/iderudit/1052609ar

DOI : https://doi.org/10.7202/1052609ar

Aller au sommaire du numéro

Éditeur(s)

Alterstice

ISSN

1923-919X (numérique)

Découvrir la revue

Citer cet article

Horgues, C. \& Scheuer, S. (2018). L'exploitation d'un corpus d'interactions en tandem anglais/français pour mieux comprendre les enjeux de la rétroaction corrective entre pairs. Alterstice, 8(1), 63-81. https://doi.org/10.7202/1052609ar

\section{Résumé de l'article}

Le tandem permet un apprentissage collaboratif où la relation entre les partenaires est moins hiérarchisée que dans les échanges entre professeur et élève en classe de langue. Chacun s'engage à faire progresser son partenaire dans sa langue et culture maternelles. Les apprentissages linguistiques et culturels y sont moins prévisibles, structurés et validés que dans le contexte institutionnel d'un cours. Les partenaires tandem accordent souvent plus d'importance à la fluidité et la convivialité de l'échange qu'à la précision des formes linguistiques. Mais qu'en est-il de la rétroaction corrective (en anglais corrective feedback), pratique clé des enseignants de langues pour favoriser le développement langagier de leurs élèves ? Les locuteurs natifs y ont-ils recours quand leur partenaire tandem produit une forme linguistique erronée ? Si oui, à quelle fréquence, quelles en sont les modalités ? Dans cet article, nous montrons comment l'exploitation d'un corpus SITAF, soit des interactions en tandem anglais-français filmées entre étudiants francophones et anglophones de l'Université Sorbonne Nouvelle, révèle que la rétroaction orale corrective en tandem face à face repose sur une dynamique co-construite mobilisant les deux partenaires et est dépendante de plusieurs variables (en particulier la L1 du correcteur, la tâche, la familiarité et la dynamique de la dyade). 


\section{7}

ARTICLE THÉMATIQUE

\section{L'exploitation d'un corpus d'interactions en tandem anglais/français pour mieux comprendre les enjeux de la rétroaction corrective entre pairs}

Céline Horgues ${ }^{1}$ et Sylwia Scheuer ${ }^{1}$

\section{Résumé}

Le tandem permet un apprentissage collaboratif où la relation entre les partenaires est moins hiérarchisée que dans les échanges entre professeur et élève en classe de langue. Chacun s'engage à faire progresser son partenaire dans sa langue et culture maternelles. Les apprentissages linguistiques et culturels y sont moins prévisibles, structurés et validés que dans le contexte institutionnel d'un cours. Les partenaires tandem accordent souvent plus d’importance à la fluidité et la convivialité de l'échange qu'à la précision des formes linguistiques. Mais qu'en est-il de la rétroaction corrective (en anglais corrective feedback), pratique clé des enseignants de langues pour favoriser le développement langagier de leurs élèves? Les locuteurs natifs y ont-ils recours quand leur partenaire tandem produit une forme linguistique erronée ? Si oui, à quelle fréquence, quelles en sont les modalités ? Dans cet article, nous montrons comment l'exploitation d'un corpus SITAF, soit des interactions en tandem anglais-français filmées entre étudiants francophones et anglophones de l'Université Sorbonne Nouvelle, révèle que la rétroaction orale corrective en tandem face à face repose sur une dynamique co-construite mobilisant les deux partenaires et est dépendante de plusieurs variables (en particulier la L1 du correcteur, la tâche, la familiarité et la dynamique de la dyade).

\section{Rattachement des auteures \\ ${ }^{1}$ Université Sorbonne Nouvelle-Paris 3, Paris, France}

\section{Correspondance}

celine.horgues@sorbonne-nouvelle.fr

\section{Mots clés}

interculturel, tandem, rétroaction corrective, apprentissage, langue étrangère

\section{Pour citer cet article}

Horgues, C. et Scheuer, S. (2018). L'exploitation d'un corpus d'interactions en tandem anglais/français pour mieux comprendre les enjeux de la rétroaction corrective entre pairs. Alterstice, 8(1), 63-82. 


\section{Introduction}

Dans les principes d'origine de la méthode tandem tels qu'ils sont décrits par Helmling (2002) et Brammerts et Calvert (2003), les partenaires sont liés par le contrat moral suivant: chaque apprenant s'engage à aider son partenaire tandem à développer ses connaissances et compétences dans sa langue maternelle (désormais L1) et sa culture maternelle. En échange, selon le principe de réciprocité, chaque apprenant s'attend à recevoir, pour la langue cible apprise (désormais L2, comprendre langue étrangère, langue seconde ou langue additionnelle selon le contexte linguistique du pays de résidence), une aide similaire apportée par son partenaire tandem.

Or la nature de la collaboration induite et de l'aide apportée par les apprenants varie en fonction des représentations qu'ils ont de leur rôle et de leur positionnement dans la dyade tandem (Tardieu et Horgues, 2015) ainsi que de l'impact possible des traditions socio-culturelles (Hofstede, 1986) et socio-éducatives (Osborn, 2009). En effet, la mission d'entraide peut être interprétée de différentes façons par les partenaires tandem : faut-il laisser parler son partenaire et favoriser la fluidité de l'expression et sa prise de confiance ou alors faut-il l'aider à améliorer la précision linguistique en corrigeant ses erreurs?

L'approche communicative de l'enseignement d'une L2 préconisée dans notre contexte consiste à favoriser le développement linguistique des apprenants en leur proposant des activités qui portent sur le sens et le contexte de l'énoncé dans une situation de communication, mais également en centrant temporairement leur attention sur la forme linguistique (importance du repérage et de l'attention, Schmidt, 1990), notamment sur la distance entre la forme erronée et la forme cible, et ce, en privilégiant la rétroaction corrective (corrective feedback, Lyster et Ranta, 1997 ou Sheen et Ellis, 2011 ; negative evidence, Gass, 2003; reactive focus-on-form instruction, Ranta et Lyster, 2018) et en exposant les apprenants au modèle des formes cibles attestées en L2 (positive evidence, Gass, 2003).

Or la configuration du tandem entre pairs représente un contexte d'apprentissage tout autre : la hiérarchie entre les participants est quasiment neutralisée par rapport aux interactions entre enseignant et apprenant, les échanges se font hors de la salle de classe et l'environnement y est donc plus détendu et amical. Les partenaires tandem sont sur un pied d'égalité dans la mesure où ils sont tour à tour experts relatifs quand ils parlent dans leur L1 et novices relatifs quand ils s'expriment en L2 (Ding, 2012). Dans ce contexte d'apprentissage particulier, les partenaires tandem ont-ils aussi recours à la rétroaction corrective pour aider leur partenaire à mieux s'approprier leur L1 ? Si oui, quelles en sont les modalités?

Pour répondre à cette interrogation, nous nous appuyons sur un corpus d'interactions orales en tandem anglais/français filmées à l'Université Sorbonne Nouvelle (France). Après avoir précisé le concept de tandem face à face et le cadre de l'étude sur la rétroaction corrective, une analyse détaillée des interactions orales va permettre de révéler les enjeux de la rétroaction corrective en tandem.

\section{État de l'art : le tandem et la rétroaction corrective}

\section{Particularités du tandem oral face à face pour l'étude de la rétroaction corrective}

Le tandem oral face à face se distingue d'autres types d'échanges interculturels dans la mesure où il place la réciprocité des apports langagiers et culturels (fournis par chaque partenaire pour sa L1) au cœur de son fonctionnement. II ne recoupe donc pas tout à fait le jumelage tel qu'il peut être pratiqué par exemple au Canada (Carignan, Deraîche et Guillot, 2015), qui valorise principalement la dimension interculturelle et ne place pas la réciprocité langagière (et la réversibilité de l'expertise linguistique) comme prérequis.

Par rapport à la télécollaboration interculturelle en ligne (Ware et O'Dowd, 2008 ; Lewis et O'Dowd, 2016), le tandem face à face possède des particularités déterminantes pour comprendre les enjeux précis de la rétroaction corrective. Les interactions orales y sont obligatoirement synchrones et donc éminemment spontanées, immédiates mais aussi éphémères. La physicalité et la réalité incarnée de l'interaction orale et en présence permettent aux partenaires tandem de prendre toute la mesure des réactions immédiates de l'interlocuteur à son 
discours (minimisation des incompréhensions linguistiques et interculturelles) et de s'appuyer sur toutes les ressources multimodales (verbales, orales et visuelles) disponibles dans la conversation pour accéder au sens et s'approprier la production des formes cibles de la L2.

\section{La rétroaction corrective}

La recherche sur la rétroaction a traditionnellement porté sur les pratiques correctives d'enseignants de langue L2 (Ellis, Loewen et Erlam, 2006 ; Gass, 2003 ; Lyster et Ranta, 1997 ; Mackey, 2006) et s'est longtemps centrée sur le contexte institutionnel où la rétroaction est dispensée en classe par l'enseignant. Dans ce cadre, les instances de correction sont définies par Lyster et Ranta (1997) comme l'intervention de l'enseignant faisant immédiatement suite à une production erronée produite par l'apprenant L2. La recherche sur les rétroactions correctives s'est alors orientée sur les domaines de la correction, la plupart du temps dans le domaine morphosyntaxique (voir Sheen, 2006) et plus rarement dans les volets sémantiques et phonologiques (voir Saito et Lyster, 2012), les stratégies de corrections effectuées (prédominance des reformulations par recast, voir Lyster, Saito et Sato, 2013), l'efficacité de ces corrections pour l'apprentissage (El Tatawy, 2002; Saito et Lyster, 2012) et leur appropriation par les apprenants (Sheen, 2006).

Plus récemment, des études se sont intéressées à la rétroaction corrective entre pairs (Lyster, Saito et Sato, 2013 ; Sato, 2017), en explorant la manière dont des élèves, apprenant tous les deux la même L2 et partageant généralement la même L1, collaborent et se corrigent en interaction dans le cadre de la classe : l'efficacité de la rétroaction entre pairs est fortement dépendante de la dynamique sociale et collaborative entre les apprenants et elle profite à la fois à celui qui est corrigé et à celui qui corrige (attention à la forme). Sato (2007) a comparé aussi des interactions entre pairs apprenant la même L2 avec des dyades apprenant-natif (L2-L1). Dans cette étude, apprenants tout comme natifs prêtaient peu d'attention à la négociation de forme, corrigeaient rarement les erreurs grammaticales et recouraient très largement aux reformulations (recasts). Ils rapportaient aussi plus d'autocorrections formelles entre pairs. Cependant il n'y avait pas de réversibilité d'expertise ni de statut induite dans les dyades de Sato (2007) : le natif et l'apprenant gardaient toujours leur statut, tout comme chez Lee (2004 et 2006). À notre connaissance, aucune étude n'a encore porté sur la rétroaction corrective par des pairs en tandem face à face. Ce contexte diffère de celui de la salle de classe en ce qu'il y a une nette asymétrie d'expertise (linguistique et culturelle) entre les partenaires tandem en fonction de qui parle sa L1 à un moment de l'interaction. Cette expertise est réversible et évolutive quand les partenaires changent de langue. Cette asymétrie temporaire et dynamique est aussi à distinguer de la hiérarchie institutionnalisée entre professeur et élèves. Les partenaires tandem n'ont d'ailleurs pas pour objectif de (ni la formation pour) se substituer à un enseignant de langues, sinon tout l'intérêt du tandem serait perdu. Enfin, les études de rétroaction portant sur les échanges en ligne ne s'intéressent en général qu'aux productions écrites asynchrones avec un focus particulier sur le domaine grammatical (voir O'Rourke, 2005; Ware et O’Dowd, 2008) et non à l'ensemble des phénomènes liés à la production orale spontanée en synchronie.

Se situant dans un entre-deux entre intervention du professionnel expert et correction collaborative entre élèves, la rétroaction corrective en tandem oral face à face pose de nombreuses questions que cet article se donne pour objectif d'explorer.

\section{Problématique et méthodologie de l'expérimentation}

\section{Questions de recherche}

Étant donné l'arrière-plan théorique sur le tandem et sur la rétroaction corrective, il nous paraît pertinent de poser les trois questions de recherche suivantes pour faire émerger la particularité de la rétroaction corrective en tandem par rapport aux études précédemment citées : 1) Les partenaires tandem se corrigent-ils ? 2) Y a-t-il un lien entre la rétroaction corrective et la L1 du correcteur ? 3) Que corrigent-ils et comment ? 


\section{Approche méthodologique générale}

Nous adaptons la méthodologie d'analyse du corrective feedback de Lyster et Ranta (1997) et Gass (2003) pour définir une instance de correction comme toute réaction verbale de l'interactant natif à une forme non conforme à la cible de la L1 produite par son partenaire tandem (L2) et visant à la mettre en conformité. Nous nous intéressons ensuite au domaine linguistique concerné par la rétroaction (lexical, grammatical ou morphosyntaxique, phonétique). Nous élargissons ici l'étude des domaines que nous avions débuté dans une étude précédente (Horgues et Scheuer, 2014) qui ne portait que sur les réatroactions correctives d'ordre phonétique car contraintes par la tâche de lecture monitorée du même corpus (parole contrôlée, non spontanée). Notre analyse des stratégies de rétroaction s'inspire également de la typologie proposée pour le domaine de l'enseignement L2 par Lyster et Ranta (1997), Sheen et Ellis (2011) ou encore Lyster, Saito et Sato (2013) et nous nous concentrons sur les catégories présentes dans les interactions en tandem observées, à savoir les reformulations $\left(\right.$ recast $\left.^{1}\right)$, les demandes de clarification et les corrections explicites ou commentaires métalinguistiques.

\section{Le protocole expérimental pour constituer le corpus SITAF}

\section{La collecte du corpus}

La collecte du corpus a été pilotée par les auteures à l'Université Sorbonne Nouvelle ${ }^{2}$. Les locuteurs et locutrices du corpus participaient au dispositif tandem anglais/français TandemP3 auquel les étudiants s'inscrivent sur la base du volontariat (les échanges et apprentissages ne font l'objet d'aucune évaluation). L'équipe enseignante propose un appariement à partir d'un questionnaire en ligne, en considérant des critères tels que le profil linguistique, l'âge, le niveau compétence L2 auto-estimé ${ }^{3}$ mais aussi les centres d'intérêts et les éventuelles requêtes particulières. Les binômes sont alors invités à prendre contact lors d'une réunion informelle où les grands principes du tandem leur sont présentés (réciprocité, autonomie, tolérance, équilibre de l'utilisation des deux langues). Ensuite, les binômes sont entièrement libres de gérer leur collaboration en tandem sur le reste du semestre universitaire.

La collecte du corpus d'interactions en tandem filmées n'a concerné que deux séances pour les 21 paires qui s'étaient portées volontaires : la première en tout début de semestre et la seconde en toute fin de semestre (trois mois plus tard). En dehors de ces deux enregistrements, les paires tandem ont conversé librement et régulièrement à une fréquence moyenne d'une fois par semaine (soit 12 rencontres au cours du semestre).

Les deux séances expérimentales filmées nous ont permis de constituer un corpus multimodal (audio et vidéo) et longitudinal d'environ 25 heures d'interaction orale, où chacune des 21 paires était composée d'une personne de langue maternelle française (les francophones natifs sont codés F01 à F21) et d'une personne de langue maternelle anglaise (les anglophones natifs sont codés A01 à A21). Un questionnaire écrit post-enregistrement (voir en annexe) a aussi permis de recueillir des métadonnées relatives au vécu et au ressenti des 42 participants vis-à-vis de leur apprentissage en tandem.

\section{Le profil des participants}

Aucun participant n'était bilingue dominant dans la langue de sa ou son partenaire. Les 21 participantes francophones (toutes des femmes) étaient étudiantes françaises de notre université. La plupart étaient anglicistes en licence d'anglais. Leur niveau général (auto-estimé) de compétence en anglais L2 atteignait 7,2/10. Les 21 participants anglophones (18 femmes et 3 hommes) étaient à l'université dans le cadre d'un séjour de mobilité internationale et provenaient de divers pays anglophones (Royaume-Uni, États-Unis, Irlande, Canada et Australie).

\footnotetext{
${ }^{1}$ Recast : reformulation simple qui consiste pour le professeur ou l'interlocuteur natif à reprendre la formulation de l'apprenant en y substituant simplement une forme correcte à la place du segment erroné.

2 Nous remercions chaleureusement les participants à l'étude, l'équipe du projet SITAF, les techniciens du studio d'enregistrement. Nous remercions aussi notre équipe de recherche (SeSylia, Prismes, EA 4398), ainsi qu'Ortolang-Ircom et le Labex EFL (ANR-10-LABX-0083) pour leur soutien financier et technique.

${ }^{3}$ Avec quatre sous composantes : compréhension et expression écrites, compréhension et expression orales.
} 
Leur niveau de compétence en français L2 était plus hétérogène que celui des francophones dans leur L2 : leur compétence langagière dépendait en grande partie de leur spécialité d'étude mais aussi de la durée de leur séjour en France au moment de l'expérimentation. Ils ont auto-estimé leur niveau général en français à $6,9 / 10^{4}$.

Les tâches de production lors des deux séances d'enregistrement

Les trois mêmes tâches collaboratives ont été réalisées par les participants lors des deux séances d'enregistrement. Les tâches se voulaient les plus ludiques et collaboratives possibles afin de réduire au maximum les effets du stress causé par les conditions du studio d'enregistrement. La consigne était simplement pour les interactants tandem de s'entraider dans la réalisation des jeux, mais aucune référence explicite à la rétroaction corrective n'a été faite (contrairement à Ware et O’Dowd, 2008).

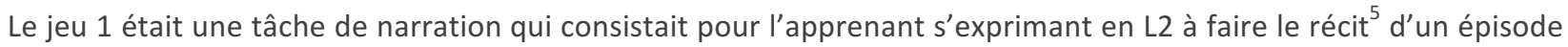
survenu pendant ses dernières vacances. II devait glisser trois mensonges dans son histoire et son interlocuteur natif devait les découvrir en lui posant des questions. La deuxième tâche était une tâche d'argumentation : le jeu 2 consistait pour les deux partenaires à débattre d'un sujet polémique soumis au moment de la discussion. Ces deux premiers jeux ont été joués dans les deux langues consécutivement (changement au bout de 15 min). La séance s'est terminée par une troisième tâche, une lecture monitorée.

\section{Principaux résultats : quelle rétroaction corrective dans le corpus de tandem anglais/français ?}

Notre analyse porte ici sur l'ensemble des séquences semi-spontanées du corpus (jeu 1 et 2, dans les deux langues lors des deux sessions d'enregistrement), ce qui représente environ 15 heures de parole. Les résultats ci-dessous permettent de répondre aux questions de recherche exposées plus haut.

Résultats généraux et discussion : la quantité de corrections

En tout, 492 interventions correctives ont été identifiées. Nous en présentons la répartition dans le tableau 1.

\section{Tableau 1. Nombre d'instances correctives classées par langue de conversation (français ou anglais, session (1 ou 2) et tâche (jeu 1 ou jeu 2)}

\begin{tabular}{|c|c|c|c|c|c|}
\hline \multirow{4}{*}{$\begin{array}{c}\text { Conversation } \\
\text { en français }\end{array}$} & \multicolumn{2}{|c|}{ session 1 } & \multicolumn{2}{c|}{ session 2 } & toutes sessions \\
\cline { 2 - 6 } & \multicolumn{2}{|c|}{201} & \multicolumn{2}{c|}{135} & 336 \\
\cline { 2 - 6 } & jeu 1 & jeu 2 & jeu 1 & jeu 2 & \\
\cline { 2 - 6 } & 158 & 43 & 95 & 40 & \\
\cline { 2 - 6 } $\begin{array}{c}\text { Conversation } \\
\text { en anglais }\end{array}$ & \multicolumn{2}{|c|}{ session 1 } & \multicolumn{2}{c|}{ session 2 } & toutes sessions \\
\cline { 2 - 7 } & jeu 1 & jeu 2 & jeu 1 & jeu 2 & 156 \\
\cline { 2 - 7 } & 69 & 24 & 47 & 16 & \\
\hline
\end{tabular}

La quantité de corrections apportées varie en fonction du type de jeu mais aussi de la session d'enregistrement. En effet, le jeu 1 de narration a suscité trois fois plus d'instances de corrections que le jeu 2 d'argumentation (voir tableau 1 ci-dessus), et ce, pour les deux profils de " correcteurs ». Ceci s'explique par le fait que la consigne du premier jeu a suscité beaucoup de rétroactions certainement car les apprenants ont essayé d'y exprimer des épisodes assez saugrenus, avec des détails très précis, ce qui les a poussés à utiliser spontanément (sans avoir accès à un dictionnaire) un lexique riche, pointu et même parfois technique (par exemple pour décrire l'eau

\footnotetext{
${ }^{4}$ La différence avec le score indiqué par les francophones n'atteint pas la significativité sur le plan statistique.

${ }^{5}$ Les partenaires avaient eu un temps de préparation de quinze minutes pour réfléchir à leur récit.
} 
" jaillissait puissamment comme un geyser ", A11). Par ailleurs, la consigne du premier jeu fixait aussi des rôles bien définis : c'était l'apprenant L2 qui avait principalement la parole et le partenaire était placé en position d'écoute afin de pouvoir ensuite poser des questions pour identifier les mensonges. II se peut que l'attention du participant natif ait été plus portée sur la forme du propos de l'apprenant que dans le jeu d'argumentation où le temps de parole et la participation à la conversation étaient plus équilibrés.

Par ailleurs, la confrontation des instances de correction en première session par rapport à la seconde session d'enregistrement (tableau 1) semble indiquer que les participants natifs tendent à corriger de moins en moins leur partenaire au fil du temps : en effet, on observe une chute de $30 \%$ du nombre de corrections réalisées par les "correcteurs" anglophones natifs en deuxième session (baisse équivalente dans les deux jeux). Si le développement langagier en L2 des partenaires au cours des 3 mois passés (pratique du tandem et exposition à l'input lors des cours de L2 ou dans la vie quotidienne) explique en partie ce résultat, nous faisons l'hypothèse, fondée sur les observations ${ }^{6}$ de Brammerts et Calvert (2003), qu'à mesure que le degré de familiarisation augmente entre les deux partenaires, la tendance à porter son attention sur la forme linguistique du propos diminue. Ils s'accoutument aux habitudes langagières et idiosyncrasies de leur interlocuteur et la rétroaction corrective passe au second plan par rapport à la fluidité et la convivialité de l'échange.

\section{L'impact de la L1 du « correcteur »}

Pour répondre à la deuxième question de recherche, nous avons comparé la quantité de rétroactions correctives observées dans les deux conditions de langues: conversations menées en français et celles en anglais par les mêmes paires tandem (tableau 1). On peut nettement constater que les francophones ont fourni deux fois plus de rétroactions correctives à leurs partenaires anglophones qu'inversement, et ce, à chaque session (respectivement 201 contre 63 en session 1 et 135 contre 63 en seconde session). La différence de niveau de compétence en L2 ${ }^{7}$ chez les deux groupes de participants n'est pas assez conséquente pour expliquer ce rapport du simple ou double dans la quantité de corrections fournies. Nous avançons qu'il faut invoquer le rôle de facteurs socioculturels pour expliquer, au moins en partie, cette observation, en particulier le rapport qu'entretiennent différentes cultures avec le concept d'autorité ou de hiérarchie entre individus. Dans la tradition des études sur les dimensions culturelles portées par Hofstede (1986), la France est ainsi décrite comme ayant une culture reposant sur une distance hiérarchique plus marquée (Large Power Distance) que celle des pays où l'anglais est langue officielle ou majoritaire (Canada, Royaume-Uni, États-Unis, Small Power Distance). Si, dans la première, les élèves s'attendraient à ce que le "maître" leur donne des directives à suivre, dans les secondes l'enseignant respecterait l'indépendance des élèves et s'attendrait à ce qu'ils trouvent eux-mêmes leurs solutions. La France et les pays anglophones y sont aussi situés à des points opposés sur l'échelle de l'évitement de l'incertitude (uncertainty avoidance) selon lequel certaines cultures (comme en France) tendraient à éviter les situations perçues comme incertaines en valorisant des codes clairs et la recherche de la vérité absolue et de précision. II ne s'agit là que de tendances très généralisantes : aucune société ne saurait se réduire à une seule culture unique, homogène et figée. Cependant, ces observations concordent bien avec la préoccupation plus manifeste des francophones du corpus envers la correction de la langue parlée.

Si la perception de la " politesse ", et plus généralement des règles de convenance sociale (en discours), ont une composante universelle, elles sont aussi fortement ancrées dans une culture donnée (Armaşu, 2012 et 2013; Brown et Levinson, 1987). À titre d'exemple, Wilkinson (2002) a rapporté aussi des réactions négatives d'étudiantes anglophones en réponse à la tendance marquée de leurs familles d'accueil françaises à corriger abondamment leurs erreurs linguistiques lors de leur séjour en immersion en France. Les anglophones de notre corpus ont

\footnotetext{
${ }^{6}$ Ils décrivaient un mécanisme d'habituation ou encore le développement d'une tolérance aux erreurs chez leurs partenaires tandem qui, au fil du temps, intervenaient de moins en moins pour rectifier leurs erreurs linguistiques.

${ }^{7}$ Les participantes francophones s'auto-estiment comme un peu meilleures en expression orale en L2 (6,8/10 contre 6,6/10) mais ne sont clairement pas deux fois plus compétentes.
} 
d'ailleurs déclaré estimer qu'il peut être impoli ou déstabilisant ${ }^{8}$ de corriger leur interlocutrice dont ils veulent " préserver la face » (voir aussi Philp, Walter et Basturkmen, 2010).

Nous faisons l'hypothèse que les Françaises de ce corpus corrigent plus fréquemment et plus naturellement car elles répliquent aussi des pratiques scolaires qui leur sont familières et qui tendent à donner une place centrale à la conformité formelle et à la correction systématique des erreurs (Osborn, 2009). L'effet "à domicile ", à savoir que les étudiantes francophones se sentaient certainement plus chez elles (c'est-à-dire dans leur pays d'origine, dans leur université), a pu également renforcer le positionnement correctif dominant adopté. Les francophones ne semblent cependant pas conscientes ${ }^{9}$ de leur positionnement "surcorrectif » ou, du moins, que leur point de référence quant à la fréquence d'intervention corrective "normale" ou attendue est différent de celui des anglophones.

\section{Le domaine de la correction}

Pour répondre à la troisième question de recherche, nous avons émis des hypothèses sur le domaine cible des rétroactions observées dans l'intégralité des séquences (en prenant en compte la nature de l'erreur produite et le contenu de la rétroaction fournie). Les corrections lexicales (aide pour trouver l'expression juste, par exemple : « la boîte de vitesse " en français ou " a psychic " en anglais) sont largement prédominantes, et ce, en particulier pour les " correcteurs " anglophones ${ }^{10}$. En effet, la moitié des corrections relèvent du domaine lexical $(52,5 \%)$, contre seulement $28 \%$ portant sur des questions de prononciation, et les points relevant de la grammaire ou de la morphosyntaxe en sont que très exceptionnellement corrigés $(12,5 \%$ de corrections grammaticales ou morphosyntaxiques et $7 \%$ combinant grammaire et lexique). Ce sont aussi des points lexicaux que les francophones ${ }^{11}$ corrigent le plus (49\%), mais leurs corrections morphosyntaxiques sont tout de même fréquentes (19\%, et $8 \%$ combinant grammaire et lexique), alors que les corrections d'ordre phonétique sont relativement minoritaires ( $15 \%$, et $9 \%$ de combinaisons comportant un aspect phonétique).

Il est intéressant de noter que les deux tâches de parole spontanée analysées ont suscité des corrections prioritairement lexicales, à la différence de la tâche de lecture analysée dans une étude précédente sur le même corpus qui a naturellement favorisé les rétroactions phonétiques (Horgues et Scheuer, 2014), et contrairement aux recherches sur des échanges écrits asynchrones où les corrections grammaticales dominent (O'Rourke, 2005 ; Ware et O'Dowd, 2008). Le format oral et synchrone des interactions ne permet pas en effet de revenir sur le détail de la correction grammaticale et focalise l'attention sur le sens véhiculé dans l'instant et les enjeux de recherche lexicale.

La relative désaffection des anglophones pour la rétroaction corrective d'ordre grammatical et morphosyntaxique peut s'expliquer par différents facteurs: a) les spécificités linguistiques de la langue française par rapport à la langue anglaise (conjugaison complexe, accords en fonction du genre, auxiliaires être/avoir, etc.), mais aussi b) les traditions éducatives ayant formé les deux profils de participants. En effet, en France, dès l'école primaire ${ }^{12}$, les enfants développent les connaissances métalinguistiques explicites nécessaires à l'analyse grammaticale de leur langue maternelle (notions de syntaxe, nature et fonction des éléments de la phrase). Ware et O'Dowd (2008) ont

\footnotetext{
${ }^{8}$ Dans le questionnaire post-enregistrement en annexes (question 25), parmi les raisons invoquées pour ne pas corriger la prononciation du partenaire, ne pas mettre mal à l'aise son partenaire (a) et ne pas être impoli (b) remportent chacune 9 votes sur 21 chez les d'anglophones, contre seulement 3 et 7 votes (respectivement) chez les francophones. Les deux groupes s'accordent sur deux raisons majoritaires: les erreurs sont trop minimes (c) et ils comprennent leur interlocteur malgré les erreurs (d).

${ }^{9}$ Dans le même questionnaire, à la question 11 «Lorsque votre partenaire fait un récit en L2, vous l'interrompez lorsque vous entendez une erreur ?», les réponses [jamais, presque jamais, parfois] remportent 14 votes sur 21 chez les francophones contre 10 sur 21 chez les anglophones.

${ }^{10}$ Pourcentages calculés sur le total des 156 rétroactions observées dans les conversations en anglais (tableau 1).

${ }_{11}^{11}$ Pourcentages calculés sur le total des 336 rétroactions observées dans les conversations en français (tableau 1).

12 Les instructions officielles (Éduscol, s. d.) préconisent 15 à 20 min quotidiennes d'étude de la langue française en cycles 2 et 3 et stipulent : " La langue doit être considérée comme un système régulier en appui sur des éléments structurés, reliés entre eux et hiérarchisés. Il est important de bien distinguer le statut de ces éléments et les fonctions syntaxiques qu'ils peuvent assumer au sein des phrases et des textes, c'est-à-dire leurs rôles possibles ».
} 
rapporté aussi une meilleure connaissance du métalangage et de la terminologie linguistique chez les participants espagnols que chez leurs partenaires américains en raison de différences socioéducatives.

\section{Prédominance et efficacité relative des reformulations (recasts)}

Pour répondre à la deuxième partie de la troisième question de recherche, nous avons classé l'ensemble des rétroactions (tableau 1) en fonction des trois catégories de stratégies correctives retenues : reformulation, demandes de clarification et enfin corrections explicites ou commentaires métalinguistiques. La stratégie de rétroaction corrective qui prédomine largement est la simple reformulation ou recast ${ }^{13}$ ( $84 \%$ en anglais et $89 \%$ en français), loin devant les deux autres catégories, qui sont représentées en proportion semblable : les demandes de clarification d'un côté $(8,3 \%$ et $5 \%$ respectivement pour chaque langue) et les corrections explicites ou commentaires métalinguistiques de l'autre $(7,7 \%$ et $6 \%)$. II n'est pas surprenant que la simple reformulation soit la stratégie de prédilection des partenaires tandem (conformément aux résultats de Sato, 2007) car elle se caractérise par sa discrétion, son aspect minimalement intrusif dans la discussion et parce la personne à qui elle est destinée ne « perd pas la face ». D’autres stratégies de rétroaction corrective utilisées par les enseignants décrites dans les recherches antérieures (Lyster, 2010 ; Lyster et Ranta, 1997 ; Lyster, Saito et Sato, 2013 ; Sheen et Ellis, 2011) ne semblent pas être utilisées dans les tandems, comme l'incitation (elicitation/prompt) ou la répétition de l'erreur, certainement en raison du manque d'input apporté (Lee, 2013) et car elles ne semblent pas appropriées (trop asymétriques et artificielles) dans une interaction entre pairs. Elles obligeraient aussi à sortir du rôle de coéquipiers et à endosser le rôle d'enseignant de langue étrangère.

Comme cela a aussi été décrit dans les travaux précédents sur la rétroaction par recast (Sato, 2007; Sheen, 2006), la fonction corrective des reformulations est parfois mal ou non saisie par les apprenants car leur saillance n'est parfois pas suffisante et qu'elles sont souvent confondues avec de simples répétitions de confirmation en conversation. Notre corpus contient plusieurs cas où les reformulations correctives des natifs ou natives semblent passer inaperçues pour les apprenants : $52,6 \%$ des rétroactions fournies en anglais et $36,9 \%$ en français ne sont pas suivies de modification de l'output par le non-natif (no uptake; résultats semblables à Sato et Lyster, 2007). Respectivement $36,5 \%$ et $52,4 \%$ des rétroactions dans les deux langues sont suivies d'une reprise totale (le restant correspondant aux reprises partielles).

Dans l'exemple ci-dessous, F01 souhaite rectifier l'erreur de conjugaison de sa partenaire anglophone (A01) mais cette dernière ne perçoit pas la fonction corrective de l'intervention (dite à voix basse et accompagnée d'un geste discret).

A01 : donc ma histoire c'est quand j'ai retourné...en Irlande... pour Noël

F01 : oui, quand tu es retournée [geste de l'index vers l'arrière = référence à un moment du passé]

A01 : oui ehm, oui j'ai retourné le le vingt-et-un de décembre pour deux semaines

F01 : [hochement de tête]

À ce propos, Sheen et Ellis (2011) ont montré que la compréhension de la fonction corrective des reformulations simples (recasts) dépendait du contexte de communication (plus facile à percevoir dans un contexte pédagogique centré sur la forme que sur le sens).

Par ailleurs, la quantité d'instances de correction apportée ne semble pas directement corrélée au niveau de compétence déclarée en L2 du partenaire ni à la " gravité de l'erreur » (impact sur l'intelligibilité du message). Dans la paire A19-F19, la locutrice francophone (F19) s'abstient clairement d'intervenir pour corriger malgré la multitude de productions erronées et produites en français, même si celles-ci sont saillantes et relativement faciles à rectifier (ex : * « on a visité à Berlin »). En revanche, d'autres participants s'attachent à fournir une rétroaction corrective

${ }^{13}$ Recast dans la typologie de Lyster et Ranta, 1997 (voir aussi Lyster, 2010; Sheen et Ellis, 2011). 
pointue sur des erreurs pouvant sembler mineures. Par exemple, F11 pointe du doigt la prononciation erronée de la syllabe finale du terme "horribLe » prononcé par son partenaire américain (A11). L'erreur n'entrave pourtant pas le sens de l'énoncé mais F11 s'assure que l'erreur est rectifiée :

A11 : c'est pas...et toutes les personnes sont français, c'est comme ...c'est comme dans le maison [geste iconique d'une grande pièce] pas juste une appartement maison [geste iconique d'une grande pièce] c'est un maison

F11 : d'accord

A11 : oh mon Dieu, c'est, c'est ouff...c'est horrib' je pense [geste de rejet]

F11 : hor-ri-BLE [doigt pointé sur chaque syllabe]

A11 : ho-ri-BLE [imite doigt pointé sur chaque syllabe]

F11 : voilà ! -BLE -BLE [surarticule en montrant exagérant le geste de la langue pour prononcer la consonne latérale]

A11 : oh parce que c'est c'est féminin ?

F11 : non parce qu'en fait tu as dit « horrib’ » tu as pas dit le « I » [rire]

A11 : horrible, ok d'accord, et....

\section{Autres résultats}

D’autres particularités de la rétroaction corrective sont liées aux caractéristiques de l'environnement en tandem oral face à face décrites en première partie.

\section{Complicité et mimétisme dans les paires tandem}

Des différences individuelles entre les paires tandem (et leur dynamique sociale, voir Sato 2017) émergent aussi : si une participante corrige beaucoup, sa partenaire tandem aura tendance - par mimétisme - à corriger abondamment aussi (par exemple, dans la paire F11-A11, une rétroaction corrective en moyenne toutes les 42 secondes en anglais et toutes les 32 secondes en français dans le jeu 1 de la première session) et inversement, une paire peut s'ancrer dans une dynamique qui consiste à laisser parler plutôt que d'interrompre pour corriger (par exemple dans la paire F19-A19, une seule correction dans chacune des deux langues pour le jeu 1, conversations d'environ $3 \mathrm{~min}$ ).

Pour les paires tandem qui se sont rencontrées très régulièrement, la complicité développée se traduit entre autres par un phénomène d'alignement correctif entre les deux partenaires, qui va jusqu'à un mimétisme physique (incluant gestes, mimiques, jeux de regards) chez les deux partenaires. Un bon exemple est la paire A11-F11, qui a développé une grande complicité ${ }^{14}$ résultant de la haute fréquence de leurs rencontres et de leur appréciation très positive de leur tandem. On peut observer un alignement gestuel quasi-parfait lors de la correction d'une séquence lexicale ou grammaticale : l'apprenant adopte spontanément le geste correctif de sa partenaire native lors de la séquence corrective (effet miroir, figure 1). Manifestation très nette de la tendance à "l'accommodation conversationnelle »(Giles et Ogay, 2007), cet alignement gestuel facilite certainement l'appropriation de la rétroaction fournie.

\footnotetext{
14 Dans le questionnaire post-enregistrement (questions 1 et 2), le tandem déclare s'être rencontré 20 fois entre les deux enregistrements à raison d'1 h ou $1 \mathrm{~h} 30$ et les deux partenaires ont évalué leur expérience en tandem par une note de 10/10 (question 17).
} 


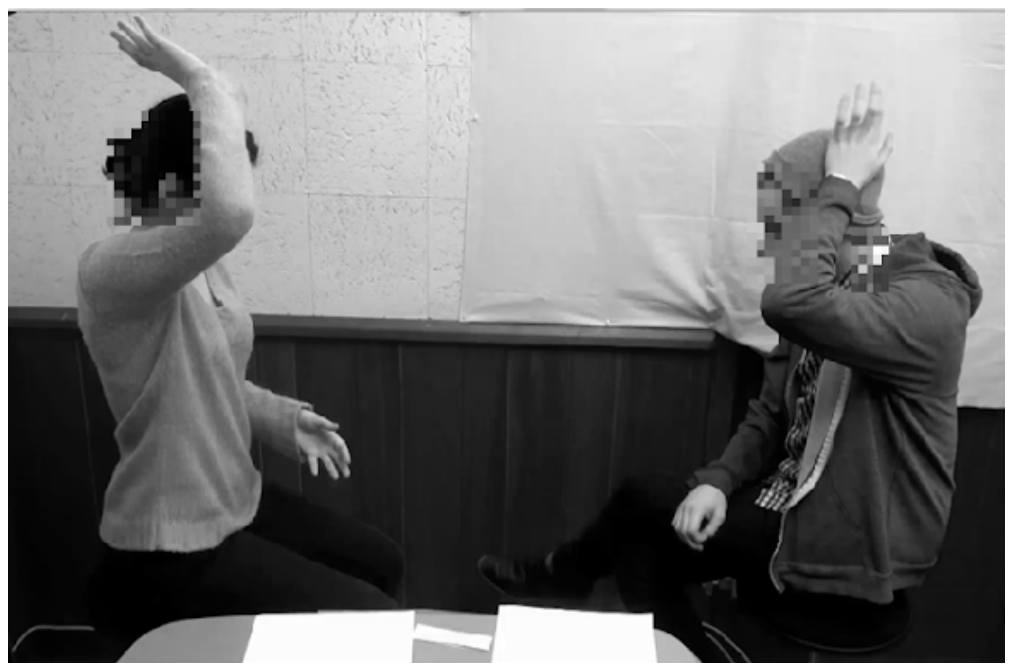

\section{Figure 1 : Alignement gestuel lors de la rétroaction corrective apportée par $\mathrm{A}^{11^{15}}$ (à droite) : lexique dans le domaine des animaux de la basse cour " rooster ", " chicken ", " hens " pour sa partenaire francophone F11 (à gauche)}

\section{Rétroaction corrective, co-construction de la séquence corrective et négociation du sens dans les échanges en tandem}

Lors d'une interaction tandem, le partenaire s'exprimant en $L 2$ est temporairement moins expert et plus novice que le locuteur natif (Ding, 2012), ce qui ne veut pas dire qu'il est cantonné au rôle passif de réception ou de simple destinataire de la rétroaction corrective. Dans de nombreuses séquences correctives du corpus, l'apprenant joue un rôle proactif aux différentes étapes de la séquence.

En effet, à l'amorce de la séquence de correction, l'apprenant sollicite souvent de manière plus ou moins explicite une rétroaction. Nous entendons par demande " explicite » une formulation verbale notifiant une demande d'aide ("c'est correct? ", "je ne sais pas le mot en français", "on peut dire X?») et nous entendons par demande " implicite » une requête plus indirecte et non verbale (hésitations, intonation montante, geste ou regard d'appel à l'autre). Par exemple, A02 combine deux stratégies de sollicitation de rétroaction lexicale: tout d'abord plus implicite par un geste, puis plus explicite par une requête verbale :

A02 : et puis j'ai pris la voiture avec quatre amis, dedans, c'était un peu [geste + mimique : étroitesse et inconfort] euh...on peut dire coincé ?

F02 : $\mathrm{mm}$ mm serré, coincé

A02 : euh chargé ?

F02 : aussi ouais, serré ?

A02 : serré [geste de pointage pour valider le terme retenu] merci, serré, et euh...on a mis trois heures dans la voiture

Une fois la rétroaction corrective fournie par le "correcteur ", l'apprenant joue aussi un rôle actif au moment de s'approprier la forme alternative proposée, au moment de la reprise (uptake, Gass, 2003), car il lui revient la responsabilité de tenir compte ou non de la proposition, de la reprendre à l'identique ou de la reprendre à son

${ }^{15}$ Conformément à l'autorisation signée par les participants du corpus, les visages ont été floutés pour conserver leur anonymat. 
compte en l'intégrant dans son propos. II arrive même parfois que l'apprenant remette en cause la rétroaction corrective fournie si cette dernière est jugée non pertinente (Storch, 2017).

Les séquences correctives observées dans les échanges tandem reposent souvent sur une co-construction (coétayage) du sens où le partenaire natif n'est pas seul responsable de la forme rectifiée. En effet, l'apprenant prend aussi part à la recherche d'une formulation plus appropriée en apportant son appréciation sur l'écart entre une formulation et l'idée qu'il souhaite exprimer.

Par exemple, c'est par une véritable séquence de négociation multimodale que la paire F13-A13 arrive à un compromis autour du choix du terme " quartier ». Pour circonscrire un domaine spatial en français, les termes de "quartier ", "ville", "village " ou "région» ont un fort ancrage culturel pour la locutrice française (F13) puisqu'en France métropolitaine une "région" correspond à une délimitation administrative précise. Son partenaire américain (A13) est conscient des différences culturelles existant entre la France et les États-Unis et des enjeux terminologiques quand il dit "mais c'est difficile parce que ce n'est pas un quartier comme à Paris ", ce qui témoigne chez lui d'une prise de conscience interculturelle (Lussier, 2011).

A13: avec quelques autres de nos potes parce que nous habitons tous plus ou moins dans... le même... région donc euh... [geste spatial de la main, suivi geste deux mains ouvertes vers le haut et mimiques bouche signalant incertitude]

F13: plus dans le même quartier? [geste spatial de la main]

A13 : ben...

F13 : parce que région c'est très grand quand même [geste spatial très ample]

A13 : ben ouais...

F13 : surtout surtout en Amérique c'est très très grand donc euh ! [rires] [geste spatial hyper ample]

A13 : ouais, je suppose c'est plus qu'un quartier, mais c'est difficile parce que ce n'est pas un quartier comme à Paris, c'est un peu plus grand, plus ... [geste spatial d'ouverture]

F13: huh, ben un peu plus grand?

A13: ouais, mais quand même

F13: d'accord, dans ta ville, quoi ? [geste spatial d'englobement]

A13: ouais, ouais, plus ou m.... [geste main paume levée vers l'autre : compromis]

F13: c'est pas un village, c'est ta ville, elle est un peu...d'accord

A13: oui, je suppose que, je suppose que ça marche [geste : compromis]

F13: oui, ça marche [+ hochement de tête d'acquiescement, valide le compromis]

\section{Conclusion}

L'analyse du corpus SITAF d'interactions en tandem anglais/français a combiné une approche qualitative et quantitative et elle a permis d'apporter un nouvel éclairage sur les particularités de la rétroaction corrective en tandem face à face entre pairs ne partageant pas la même langue maternelle. Différentes variables influencent la rétroaction corrective par les partenaires tandem : la L1 du " correcteur », le domaine de correction, la stratégie de correction, le temps de familiarisation, la tâche de production, la compétence perçue du partenaire en L2 et des différences individuelles ayant trait à la dynamique de la dyade et à des phénomènes d'accommodation. 
Cette expérimentation nous a aussi permis de souligner le rôle des traditions éducatives et culturelles pour rendre compte du profil correctif des locuteurs en tandem, mais elle nous a aussi confronté aux limites de la notion même de rétroaction corrective prise en charge par l'interactant supposément plus compétent (natif), puisqu'on observe souvent un processus de co-construction du discours auquel les deux partenaires prennent activement part et qui reflète bien le fondement collaboratif du tandem où les rapports d'expertise (linguistique/culturelle) sont éminemment dynamiques, réversibles et évolutifs (voir Kramsch, 2003).

La rétroaction corrective en tandem a pourtant ses limites : elle n'est pas aussi experte ou systématique que celle qui est dispensée par un professionnel de l'enseignement des langues. Ce n'est d'ailleurs pas ce que l'on attend des partenaires tandem. Beaucoup d'erreurs ne sont pas corrigées, en particulier quand il y a un fort degré de familiarisation entre les deux partenaires, mais cette tolérance aux erreurs a aussi des avantages puisqu'elle représente des avantages psycho-affectifs (empathie, confiance, réduction de l'anxiété à s'exprimer en L2, motivation accrue, voir Dörnyei, 2009 et Sato et Lyster, 2012) qui sont des atouts indéniables pour le développement des compétences interactionnelles et interculturelles. Par ailleurs, comme dans le cas de la rétroaction fournie par l'enseignant ou en salle de classe (Ellis, Loewen et Erlam, 2006 ; Gass, 2003 ; Lyster et Ranta, 1997 ; Mackey, 2006), il arrive également qu'une erreur soit relevée mais pas rectifiée dans le tour de parole de l'apprenant (uptake). Or le simple fait que l'attention de l'apprenant soit attirée sur un différentiel de formes rend ce type d'interaction potentiellement riche pour le développement de la L2. En effet, dans les théories du repérage et ou de l'attention portée à la forme (Noticing Hypothesis et Attention Theory, Gass, 2003 ; Lyster et Ranta, 1997 ; Mackey, 2006 ; Schmidt, 1990), le seul fait que l'apprenant soit amené à constater un écart entre la forme utilisée (output) et la forme cible (target) est positif. L'absence de reprise (uptake, réaction verbalisée de l'apprenant) et de rectification immédiate ne signifient pas nécessairement un échec en termes d'apprentissage (Lyster, 1998). Cependant, dans la perspective interactionniste, un travail de négociation sur la forme et le sens co-construit par les partenaires tandem sera le gage d'un apprentissage langagier plus profond (Long, 2007 ; Swain et Lapkin, 1998).

Notre analyse de la rétroaction corrective dans le corpus SITAF nous mène à faire quelques suggestions pour des dispositifs tandem auprès d'étudiants et de jeunes adultes : il pourrait être utile de réfléchir à une formation des binômes tandem aux enjeux de la sollicitation et de l'apport correctif (co-construction de la séquence corrective

plutôt que relation unilatérale, prise de conscience des traditions et attentes culturelles et éducatives, stratégies possibles) que les apprenants en tandem pourraient ensuite s'approprier en fonction de leur sensibilité et de leur culture individuelles.

\section{Références bibliographiques}

Armaşu, V. D. (2012). Modern approaches to politeness theory. A cultural context. Lingua, Language and Culture, 11(1), 9-19.

Armaşu, V. D. (2013). Intercultural Competence and Politeness Theory. Language and Culture, 12(1), 31-39.

Brammerts, H. et Calvert, M. (2003). Learning by communicating in tandem. Dans T. Lewis et L. Walker (dir.), Autonomous language learning in tandem (p. 45-59). Sheffield : Academy Electronic Press.

Brown, P. et Levinson, S. C. (1987). Politeness: Some universals in language usage. Studies in interactional sociolinguistics. Cambridge : Cambridge University Press.

Carignan, N., Deraîche, M. et Guillot, M. C. (2015). Jumelages interculturels, communication, inclusion et intégration. Montréal : Presses de l'Université du Québec.

Ding, A. (2012). Native speaker/nonnative speaker interaction and orientation to novice/expert identity. Journal of Pragmatics, 44, 1503-1518.

Dörnyei, Z. (2009). The psychology of second language acquisition. Oxford : Oxford University Press.

Éduscol (s.d.). Ressources d'accompagnement du programme de français aux cycles 2 et 3 : étude de la langue. [programmes et instructions officielles en français pour l'école primaire en France]. http://eduscol.education.fr/cid106031/ressources-francais-etude-langue.html [mise à jour en juillet 2017, consultation le 25 juin 2018] 
Ellis, R., Loewen, S. et Erlam, R. (2006). Implicit and explicit corrective feedback and the acquisition of L2 grammar. Studies in Second Language Acquisition, 28, 339-368.

El Tatawy, M. (2002). Corrective feedback in second language acquisition. Working Papers in TESOL \& Applied Linguistics, Teachers' College, Columbia University, 1-19.

Gass, S. M. (2003). Input and interaction. Dans C. J. Doughty et M. H. Long (dir.), The handbook of second language acquisition (p. 224-255). Oxford : Blackwell.

Giles, H. et Ogay, T. (2007). Communication Accommodation Theory. Dans C. B. Whaley et W. Samter (dir.), Explaining communication. Contemporary theories and exemplars (p. 326-344). Mahwah, NJ : Lawrence Erlbaum Associates.

Hofstede, G. (1986). Cultural differences in teaching and learning. International Journal of Intercultural Relations, 10, 301-320.

Horgues, C. et Scheuer, S. (2015). Why some things are better done in tandem? Dans J. A. Mompeán et J. FouzGonzález (dir.), Investigating English pronunciation: Current trends and directions (p. 47-82). Basingstoke and New York : Palgrave Macmillan.

Horgues, C. et Scheuer S. (2014). «I understood you, but there was this pronunciation thing... ": L2 pronunciation feedback in English/French tandem interactions. Research in Language 12(2), 145-161.

Helmling, B. (2002). L'apprentissage autonome des langues en tandem. Paris : Didier.

Kramsch, C. (2003). The privilege of the non-native speaker. Dans C. Blyth (dir.), The sociolinguistics of foreignlanguage classrooms: Contributions of the native, the near-native, and the non-native speaker (p. 251-262). Boston, MA : Heinle.

Lee, E. (2013). Corrective feedback preferences and learner repair among advanced ESL students. System, 41(2), 217-230.

Lee, L. (2004). Learners' perspectives on networked collaborative interaction with native speakers of Spanish in the U.S. Language Learning \& Technology, 8(1), 83-100.

Lee, L. (2006). A study of native and nonnative speakers' feedback and responses in Spanish-American networked collaborative interactions. Dans J. Belz et S. Thorne (dir.), Internet-mediated intercultural foreign language education (p. 147-176). Boston : Heinle and Heinle.

Long, M. H. (2007). Problems in SLA. Mahwah, NJ : Lawrence Erlbaum.

Lewis, T. et O’Dowd, R. (2016). Online intercultural exchange: Policy, pedagogy, practice. London : Routledge.

Lussier, D. (2011). Language, thought and culture: Links to intercultural communicative competence. Canadian and International Education / Education canadienne et internationale, 40(2), 34-60.

Lyster, R. (1998). Form in immersion classroom discourse: In or out of focus?. Canadian Journal of Applied Linguistics (CJAL) / Revue Canadienne de Linguistique Appliquée (RCLA), 1(1-2), 53-82.

Lyster, R. (2010). Enseignement centré sur la forme et acquisition du genre grammatical en français L2. Canadian Journal of Applied Linguistics (CJAL) / Revue Canadienne de Linguistique Appliquée (RCLA), 13(2), 73-93.

Lyster, R. et Ranta, L. (1997). Corrective feedback and learner uptake. Studies in Second Language Acquisition, 19(1), 37-66.

Lyster, R., Saito, K. et Sato, M. (2013). Oral corrective feedback in second language classrooms. Language Teaching, 46, 1-40.

Mackey, A. (2006). Feedback, noticing and instructed second language learning. Applied Linguistics, 27(3), 405-430.

O'Rourke, B. (2005). Form-focused interaction in online tandem learning. CALICO Journal, 2(3), 433-466.

Alterstice - Revue Internationale de la Recherche Interculturelle, vol. 8, $n^{\circ} 1$ 
Osborn, M. ( 2009). Être élève en Angleterre et en France. Revue internationale d'éducation de Sèvres, 50, 87-98. https://journals.openedition.org/ries/521\#text

Philp, J., Walter, S. et Basturkmen, H. (2010). Peer interaction in the foreign language classroom: What factors foster a focus on form? Language Awareness, 19(4), 261-279.

Ranta, L., et Lyster, R. (2018). Form-focused Instruction. Dans P. Garrett et J. M. Cots (dir.), The Routledge handbook of language awareness (p. 40-56). New York : Routledge. https://www.routledgehandbooks.com/doi/10.4324/9781315676494.ch4

Saito, K. et Lyster, R. (2012). Investigating the pedagogical potential of recasts for L2 vowel acquisition. TESOL Quarterly, 46(2), 387-398.

Sato, M. (2007). Social relationships in conversational interaction: Comparison of learner-learner and learner-NS dyads. Japan Association for Language Teaching, 29(2), 183-208.

Sato, M. (2017). Oral peer corrective feedback. Dans H. Nassaji et E. Kartchava (dir.), Corrective feedback in second language teaching and learning. Research, theory, applications, implications (p. 19-34). New York/London : Routledge.

Sato, M. et Lyster, R. (2007). Modified output of Japanese EFL learners: Variable effects of interlocutor vs. feedback types. Dans A. Mackey (dir.), Conversational interaction in Second Language Acquisition: A collection of empirical studies (p. 123-142). Oxford : Oxford University Press.

Sato, M. et Lyster, R. (2012). Peer interaction and corrective feedback for accuracy and fluency development. Studies in Second Language Acquisition, 54, 591-686.

Schmidt, R. (1990). The role of consciousness in second language learning. Applied Linguistics, 11(2), 129-58.

Sheen, Y. (2006). Exploring the relationship between characteristics of recasts and learner uptake. Language Teaching Research, 10(4), 361-392.

Sheen, Y. et Ellis, R. (2011). Corrective feedback in language teaching. Dans E. Hinkel (dir.), The Handbook of research in second language teaching and learning, vol. 2 (p. 593-610). New York: Routledge.

Storch, N. (2017). Sociocultural theory in the L2 classroom. Dans S. Loewen et M. Sato (dir.), The Handbook of instructed second language acquisition (p. 69-84). New York : Routledge.

Swain, M. et Lapkin, S. (1998). Interaction and second language learning: Two adolescent French immersion students working together. The Modern Language Journal, 82(3), 320-337.

Tardieu, C. et Horgues, C. (2015). Positionnement correctif dans les interactions orales en tandem anglais-français : aspects de la correction du lexique, de la grammaire et de la phonologie. Communication présentée au colloque ACEDLE 2015, Université Lumière Lyon 2, Lyon.

Ware, P. D. et O'Dowd, R. (2008). Peer feedback on language form in telecollaboration. Language learning and technology, 12(1), 43-63.

Wilkinson, S. (2002). The omnipresent classroom during summer study abroad: American students in conversation with their French hosts. The Modern Language Journal, 86, 157-173. 


\section{Annexe}

\section{Questionnaire bilan général tandem (version pour les francophones)}

Niveau d'étude :

Spécialité :

1. Nombre de rencontres en tandem depuis la réunion du 31 janvier $2013: \ldots \ldots \ldots$

Ce nombre de rencontres vous a semblé (entourez la réponse) :

insuffisant /satisfaisant / trop important

2. La fréquence des rencontres avec votre partenaire tandem (entourez la réponse) :

2 fois par semaine / 1 fois par semaine / 1 fois toutes les deux semaines / 1 fois tous les 20 jours/ 1 fois par mois/

Vous avez trouvé que la fréquence des conversations était : insuffisante /satisfaisante / trop fréquente

3. En moyenne, vos rencontres tandem durent :

30 mins / $1 \mathrm{~h} / 1 \mathrm{~h} 30$ / 2 heures / plus de deux heures

4. À combien estimez-vous le pourcentage global de temps passé à parler en français pendant vos conversations tandems : $[0,10,20,30,40,50,60,70,80,90,100] \%$ du temps

5. À combien estimez-vous le pourcentage global de temps passé à parler en anglais pendant vos conversations tandems : $[0,10,20,30,40,50,60,70,80,90,100] \%$ du temps

6. Au cours du semestre, avez-vous l'occasion de converser avec d'autres anglophones : quotidiennement/plusieurs fois par semaine/une fois par semaine/à quelques reprises dans le semestre/ non

Précisez:

7. Lors des conversations avec votre partenaire tandem :

\begin{tabular}{|c|c|c|c|c|c|c|}
\hline & toujours & $\begin{array}{l}\text { presque } \\
\text { toujours }\end{array}$ & souvent & parfois & $\begin{array}{l}\text { presque } \\
\text { jamais }\end{array}$ & jamais \\
\hline $\begin{array}{l}\text { Vous commencez à parler dans la même langue à } \\
\text { chaque rencontre (quelle langue: ) }\end{array}$ & & & & & & \\
\hline $\begin{array}{l}\text { Vous parlez d'abord dans une langue pendant le début } \\
\text { de la conversation puis passez ensuite à l'autre langue }\end{array}$ & & & & & & \\
\hline $\begin{array}{l}\text { Vous parlez l'essentiel du temps dans une des deux } \\
\text { langues }\end{array}$ & & & & & & \\
\hline $\begin{array}{l}\text { Vous passez une séance à parler une langue puis } \\
\text { alternez à la séance suivante }\end{array}$ & & & & & & \\
\hline $\begin{array}{l}\text { Chacun passe d'une langue à l'autre assez souvent } \\
\text { (par exemple quand un problème d'expression se } \\
\text { pose) }\end{array}$ & & & & & & \\
\hline $\begin{array}{l}\text { Vous mélangez les deux langues pour } \\
\text { expliquer/demander du vocabulaire, de la grammaire }\end{array}$ & & & & & & \\
\hline Autre (précisez) : & & & & & & \\
\hline
\end{tabular}


8. Lors d'une conversation en langue étrangère, si vous avez un doute sur l'expression (grammaire, vocabulaire, phonétique) :

\begin{tabular}{|l|l|l|l|l|l|l|}
\hline & toujours & $\begin{array}{l}\text { presque } \\
\text { toujours }\end{array}$ & souvent & parfois & $\begin{array}{l}\text { presque } \\
\text { jamais }\end{array}$ & jamais \\
\hline Vous expliquez votre problème en langue étrangère & & & & & \\
\hline $\begin{array}{l}\text { Vous expliquez votre problème dans votre langue } \\
\text { maternelle }\end{array}$ & & & & & & \\
\hline $\begin{array}{l}\text { Vous vous exprimez comme si de rien n'était et } \\
\text { attendez une éventuelle réaction du partenaire }\end{array}$ & & & & & & \\
\hline
\end{tabular}

9. Lorsque vous faites une erreur en anglais :

\begin{tabular}{|l|l|l|l|l|l|l|}
\hline & toujours & $\begin{array}{l}\text { presque } \\
\text { toujours }\end{array}$ & souvent & parfois & $\begin{array}{l}\text { presque } \\
\text { jamais }\end{array}$ & \begin{tabular}{l} 
jamais \\
\hline Votre partenaire tandem vous corrige
\end{tabular} \\
\hline Il/elle corrige votre vocabulaire & & & & & & \\
\hline II/elle corrige votre grammaire & & & & & & \\
\hline II/elle corrige votre prononciation & & & & & & \\
\hline
\end{tabular}

10. Lorsque votre partenaire tandem fait une erreur en français :

\begin{tabular}{|l|l|l|l|l|l|l|}
\hline & toujours & $\begin{array}{l}\text { presque } \\
\text { toujours }\end{array}$ & souvent & parfois & $\begin{array}{l}\text { presque } \\
\text { jamais }\end{array}$ & jamais \\
\hline Vous le corrigez & & & & & & \\
\hline Vous corrigez son vocabulaire & & & & & & \\
\hline Vous corrigez sa grammaire & & & & & & \\
\hline Vous corrigez sa prononciation & & & & & & \\
\hline
\end{tabular}

11. Lorsque votre partenaire fait un récit en français

\begin{tabular}{|l|l|l|l|l|l|l|}
\hline & toujours & $\begin{array}{l}\text { presque } \\
\text { toujours }\end{array}$ & souvent & parfois & $\begin{array}{l}\text { presque } \\
\text { jamais }\end{array}$ & jamais \\
\hline Vous l'écoutez et essayez de ne pas l'interrompre & & & & & & \\
\hline $\begin{array}{l}\text { Vous l'écoutez et posez régulièrement des questions } \\
\text { pour l'aider à poursuivre }\end{array}$ & & & & & & \\
\hline $\begin{array}{l}\text { Vous l'interrompez lorsque l'expression est difficile à } \\
\text { comprendre }\end{array}$ & & & & & & \\
\hline Vous l'interrompez lorsque vous entendez une erreur & & & & & & \\
\hline
\end{tabular}

12. Avez-vous noté chez votre partenaire des différences dans la manière de mener une conversation qui seraient liées à sa culture d'origine ? OUI/NON

Si oui, expliquez :

\section{Avez-vous aidé votre partenaire dans son travail universitaire ? OUI/NON}

14. Votre partenaire vous a-t-il aidé.e. dans votre travail universitaire ? OUI/NON

15. Le travail en tandem vous a permis d'améliorer vos compétences (entourez)

\begin{tabular}{|c|c|c|c|c|c|c|}
\hline - culturelles & 0 & 1 & 2 & 3 & 4 & 5 \\
\hline - phonétiques & 0 & 1 & 2 & 3 & 4 & 5 \\
\hline - grammaticales & 0 & 1 & 2 & 3 & 4 & 5 \\
\hline - lexicales & 0 & 1 & 2 & 3 & 4 & 5 \\
\hline - en aisance générale & 0 & 1 & 2 & 3 & 4 & 5 \\
\hline
\end{tabular}

16. Évaluez votre confiance pour parler en anglais avant de commencer le tandem et après :

\begin{tabular}{|c|c|c|c|c|c|c|c|c|c|c|}
\hline \multirow[b]{2}{*}{ avant : 0} & \multicolumn{10}{|c|}{$(0=-$ confiant, $10=+$ confiant $)$} \\
\hline & 1 & 2 & 3 & 4 & 5 & 6 & 7 & 8 & 9 & 10 \\
\hline après : 0 & 1 & 2 & 3 & 4 & 5 & 6 & 7 & 8 & 9 & 10 \\
\hline
\end{tabular}


17- Notez sur une échelle de 0 à 10, votre expérience de travail en tandem (appréciation globale)

\begin{tabular}{|lllllllllll|}
\hline \multicolumn{1}{l}{0 : le plus négatif } & \multicolumn{1}{l}{10 : le plus positif } \\
\hline 0 & 1 & 2 & 3 & 4 & 5 & 6 & 7 & 8 & 9 & 10 \\
\hline
\end{tabular}

18- Quelle est votre appréciation globale sur les conversations en tandem ? (obstacles rencontrés, bénéfices tirés)

Questionnaire bilan : le tandem et la prononciation (version pour les francophones)

\section{Quand vous parlez avec votre partenaire tandem en français :}

19. Est-ce que vous adaptez votre façon de parler quand vous vous adressez à votre partenaire : OUI/NON et comment :

\begin{tabular}{|l|l|l|}
\hline & OUI & NON \\
\hline Je parle plus lentement & & \\
\hline J'articule plus clairement & & \\
\hline Je parle plus fort & & \\
\hline J'utilise une intonation plus claire & & \\
\hline J'utilise un vocabulaire plus simple & & \\
\hline J'utilise une construction de phrase plus simple & & \\
\hline Je fais des phrases plus courtes & & \\
\hline
\end{tabular}

20. Diriez-vous que l'accent de votre partenaire tandem en français est : (entourez) très fort / assez fort / moyen / assez léger / léger/ très léger / pas d'accent du tout

21. Que pensez-vous de l'accent anglais en français en général :

22. Je corrige la prononciation de mon partenaire (entourez) :

systématiquement quand je note une erreur / presque toujours / souvent / parfois / seulement quand il- elle me le demande / seulement quand il- elle me pose une question précise sur un mot / très rarement / jamais

23. Je préfère corriger la prononciation de mon partenaire (entourez) :

au moment même où je perçois une erreur / à la fin de sa phrase / quand il ou elle a fini d'exprimer toute son idée / en fin de séance tandem

24. Que corrigez-vous dans la prononciation de votre partenaire :

\begin{tabular}{|l|l|l|l|}
\hline & OUI & NON & Donnez quelques exemples \\
\hline Son intonation & & & \\
\hline Son rythme & & & \\
\hline Son accentuation & & & \\
\hline Son débit & & & \\
\hline Ses consonnes & & & \\
\hline Ses voyelles & & & \\
\hline Son aisance en général & & & \\
\hline
\end{tabular}

Autres : 
25. Les moments où vous ne corrigez pas la prononciation de votre partenaire, c'est parce que :

\begin{tabular}{|l|l|l|}
\hline & OUI & NON \\
\hline Les erreurs sont trop minimes & & \\
\hline Il y aurait trop d'erreurs à corriger & & \\
\hline Je ne veux pas mettre mal à l'aise mon partenaire & & \\
\hline Je ne veux pas interrompre son idée & & \\
\hline Je trouve impoli de le corriger ou de l'interrompre & & \\
\hline Je n'apprécie pas moi-même qu'on me corrige & & \\
\hline Je ne pense pas que la correction serait utile & & \\
\hline Je le/la comprends malgré ses erreurs de prononciation & & \\
\hline Mon partenaire ne prend pas en compte mes corrections & & \\
\hline Je ne perçois pas ses erreurs & & \\
\hline
\end{tabular}

26. La prononciation de votre partenaire en français vous empêche de le/la comprendre en français dans ?.... des cas :

\begin{tabular}{|lllllllllll|}
\hline 0 & $10 \%$ & $20 \%$ & $30 \%$ & $40 \%$ & $50 \%$ & $60 \%$ & $70 \%$ & $80 \%$ & $90 \%$ & $100 \%$ \\
\hline
\end{tabular}

Donnez des exemples précis dont vous vous souvenez :

27. Les conseils que vous avez apportés à mon partenaire lui ont permis d'améliorer sa prononciation :

Je n'ai pas remarqué/ oui, presque tous / sur certains points seulement / non pas vraiment / absolument pas

Points améliorés : problèmes qui persistent :

28. Quels sont les problèmes de prononciation en français chez les anglophones que vous trouvez ennuyeux même s'ils ne gênent pas la compréhension?

Quand vous parlez avec votre partenaire tandem en anglais :

29. Diriez-vous que votre accent en anglais est (entourez) : très fort / assez fort / moyen / assez léger / léger / très léger / pas d'accent du tout

30. Que pensez-vous de l'accent français en anglais en général :

31. Quels points pensez-vous devoir améliorer pour la prononciation de l'anglais :

32. Votre partenaire corrige votre prononciation (entourez): systématiquement quand il ou elle note une erreur / presque toujours / souvent / parfois / seulement quand je le lui demande / seulement quand je lui pose une question précise sur un mot / très rarement / jamais

33. Mon partenaire semble préférer corriger ma prononciation (entourez) : au moment même où il ou elle perçoit une erreur / à la fin de ma phrase / quand j'ai fini d'exprimer toute son idée / en fin de séance tandem 
34. Que corrige votre partenaire dans votre prononciation en anglais :

\begin{tabular}{|l|l|l|l|}
\hline & OUI & NON & Donnez quelques exemples \\
\hline Mon intonation & & & \\
\hline Mon rythme & & & \\
\hline Mon accentuation & & & \\
\hline Mon débit & & & \\
\hline Mes consonnes & & & \\
\hline Mes voyelles & & & \\
\hline $\begin{array}{l}\text { Mon aisance en } \\
\text { général }\end{array}$ & & & \\
\hline
\end{tabular}

Autres :

35. Trouvez-vous que votre partenaire corrige votre prononciation :

\begin{tabular}{|l|l|l|}
\hline & OUI & NON \\
\hline Trop souvent, cela coupe notre conversation & & \\
\hline Trop souvent, cela me met mal à l'aise & & \\
\hline Très souvent mais c'est utile & & \\
\hline De façon modérée et cela me convient & & \\
\hline Rarement, c'est dommage & & \\
\hline Rarement mais je préfère parler sans être interrompu & & \\
\hline
\end{tabular}

36. Votre prononciation en anglais vous empêche d'être compris par votre partenaire dans ?..des cas$$
10 \%
$$$$
20 \% \quad 30 \%
$$$$
40 \%
$$

$50 \%$

$60 \%$

$70 \%$

$80 \%$

$90 \% \quad 100 \%$

Donnez des exemples précis :

37. Les conversations avec votre partenaire et ses conseils vous ont permis d'améliorer vos problèmes de prononciation :

Je n'ai pas remarqué/ oui, presque tous / sur certains points seulement / non pas vraiment / absolument pas

Points améliorés :

...problèmes qui persistent : 